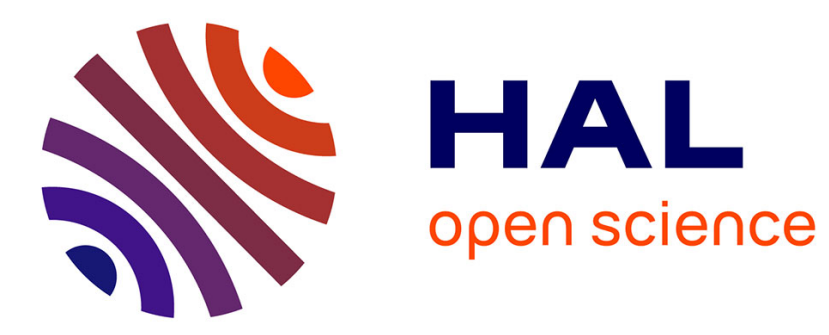

\title{
In-cavity pulse shaping by spectral sculpturing in mode-locked fibre lasers
}

Sonia Boscolo, Christophe Finot, Junsong Peng

\section{To cite this version:}

Sonia Boscolo, Christophe Finot, Junsong Peng. In-cavity pulse shaping by spectral sculpturing in mode-locked fibre lasers. 18th International Conference on Transparent Optical Network (ICTON), Jul 2016, Trento, Italy. 10.1109/ICTON.2016.7550379 hal-01312243

\section{HAL Id: hal-01312243 \\ https://hal.science/hal-01312243}

Submitted on 14 May 2016

HAL is a multi-disciplinary open access archive for the deposit and dissemination of scientific research documents, whether they are published or not. The documents may come from teaching and research institutions in France or abroad, or from public or private research centers.
L'archive ouverte pluridisciplinaire HAL, est destinée au dépôt et à la diffusion de documents scientifiques de niveau recherche, publiés ou non, émanant des établissements d'enseignement et de recherche français ou étrangers, des laboratoires publics ou privés. 


\title{
In-Cavity Pulse Shaping by Spectral Sculpturing in Mode-Locked Fibre Lasers
}

\author{
Sonia Boscolo, Junsong Peng, and Christophe Finot* \\ Aston Institute of Photonic Technologies, Aston University, Birmingham B4 7ET, United Kingdom \\ * Laboratoire Interdisciplinaire Carnot Bourgogne, UMR 6303 UBFC-CNRS, 2178 Dijon, France \\ E-mail: s.a.boscolo@aston.ac.uk
}

\begin{abstract}
We review our recent progress on the realisation of pulse shaping in passively-mode-locked fibre lasers by inclusion of an amplitude and/or phase spectral filter into the laser cavity. We numerically show that depending on the amplitude transfer function of the in-cavity filter, various advanced temporal waveforms can be generated, including parabolic, flattop and triangular pulses. An application of this approach using a flattop spectral filter is shown to achieve the direct generation of high-quality sinc-shaped optical Nyquist pulses with a widely tunable bandwidth from the laser oscillator. We also present the operation of an ultrafast fibre laser in which conventional, dispersion-managed and dissipative soliton mode-locking regimes can be selectively and reliably targeted by adaptively changing the dispersion profile and bandwidth programmed on an in-cavity programmable filter.
\end{abstract}

Keywords: mode-locked fibre lasers, nonlinear pulse shaping, frequency filtering, nonlinear fibre optics.

\section{INTRODUCTION}

At the level of fundamental research, mode-locked lasers constitute an ideal platform for the investigation of original and complex nonlinear dynamics of ultrashort pulses, while at the applied research level, pulses with different and optimised features - e.g., in terms of pulse duration, temporal and/or spectral shape, width, energy, repetition rate and emission bandwidth - are sought with the general constraint of developing efficient cavity architectures. In these two aspects, fibre laser cavities, which offer compact and versatile design, represent an area of intense development. Recently, the inclusion of a spectral pulse shaper into the cavity of a mode-locked fibre laser has emerged as a method to achieve a potentially high degree of control over the dynamics and the output of the laser [1-3], while obviously entailing a more power efficient technique than pulse shaping implemented through direct filtering of a laser output. The use of a spectral filter based on a Fourier-domain programmable optical processor [4], when placed inside a laser cavity, has the potential to allow the operation of lasers that exhibit pulse characteristics that can be controlled purely through software control. In this paper, we review our recent results and advances in the area, by numerically describing a novel approach to achieving different regimes of pulse generation in a passively-mode-locked fibre laser, which relies on nonlinear in-cavity pulse dynamics and pulse shaping by an in-cavity amplitude and phase spectral filter [5]. By changing the amplitude profile applied on the filter, we are able to create various output temporal waveforms of fundamental and practical interest, ranging from bright and dark parabolic profiles to a flat-top profile and triangular and sawtooth (asymmetric triangular) profiles. As an application of this general approach, we show that the use of an incavity flat-top spectral filter makes it possible to directly generate sinc-shaped Nyquist pulses of high quality and of a widely-tunable bandwidth from the laser [6]. We also report on a recently-developed versatile erbium-doped fibre (EDF) laser, in which conventional soliton, dispersion-managed (DM) soliton (stretched-pulse) and dissipative soliton (DS) mode-locking regimes can be selectively and reliably targeted by programming different group-velocity dispersion (GVD) profiles and bandwidths on an in-cavity programmable filter [7].

\section{IN-CAVITY PULSE SHAPING}

The design of in-cavity pulse shaping by spectral filtering exploits the understanding gained by our recent work [5]. The cavity configuration considered is a simple ring cavity (left panel of Fig. 1), comprising a short (a few meters) segment of rare-earth doped fibre with normal GVD that acts as the gain and nonlinear element of the cavity, a saturable absorber (SA) element initiating and stabilising the mode-locked operation, and an amplitude and phase spectral filter, which realises the pulse shaping. Numerical simulations of the laser are based on a nondistributed model solving pulse propagation within the fibre section by a standard modified nonlinear Schrödinger equation and including the discrete action of the SA, output coupler and spectral filter. The filter is modelled by the spectral response $H(f)=R(f) \exp \left[i \beta_{2 \text {,acc }}(2 \pi f)^{2} / 2\right]$, where $R(f)$ is the Fourier transform of a certain objective waveform function $r(t)$, and the spectral phase adds a specific amount of GVD $\beta_{2, \text { acc }}$ to the cavity, which permits to control the net cavity dispersion. The main pulse-shaping mechanism of our laser design relies on enhanced spectral filtering of a nonlinearly spectrally broadened pulse in the cavity [5]. A significant nonlinear phase shift is impressed on the pulse in the fibre section. The spectral filter compensates both the broad pulse duration and bandwidth after the fibre segment. Because the nonlinearly broadened spectrum of the pulse at the output of the fibre is wider than the filter's spectral response and fairly flat within the filter bandwidth, it 
can be sliced without caring for the details of its structure. This is the enabling mechanism for the formation of pulses of the desired temporal shape at the output of the filter, impressed on the pulse by the filter's impulse response.
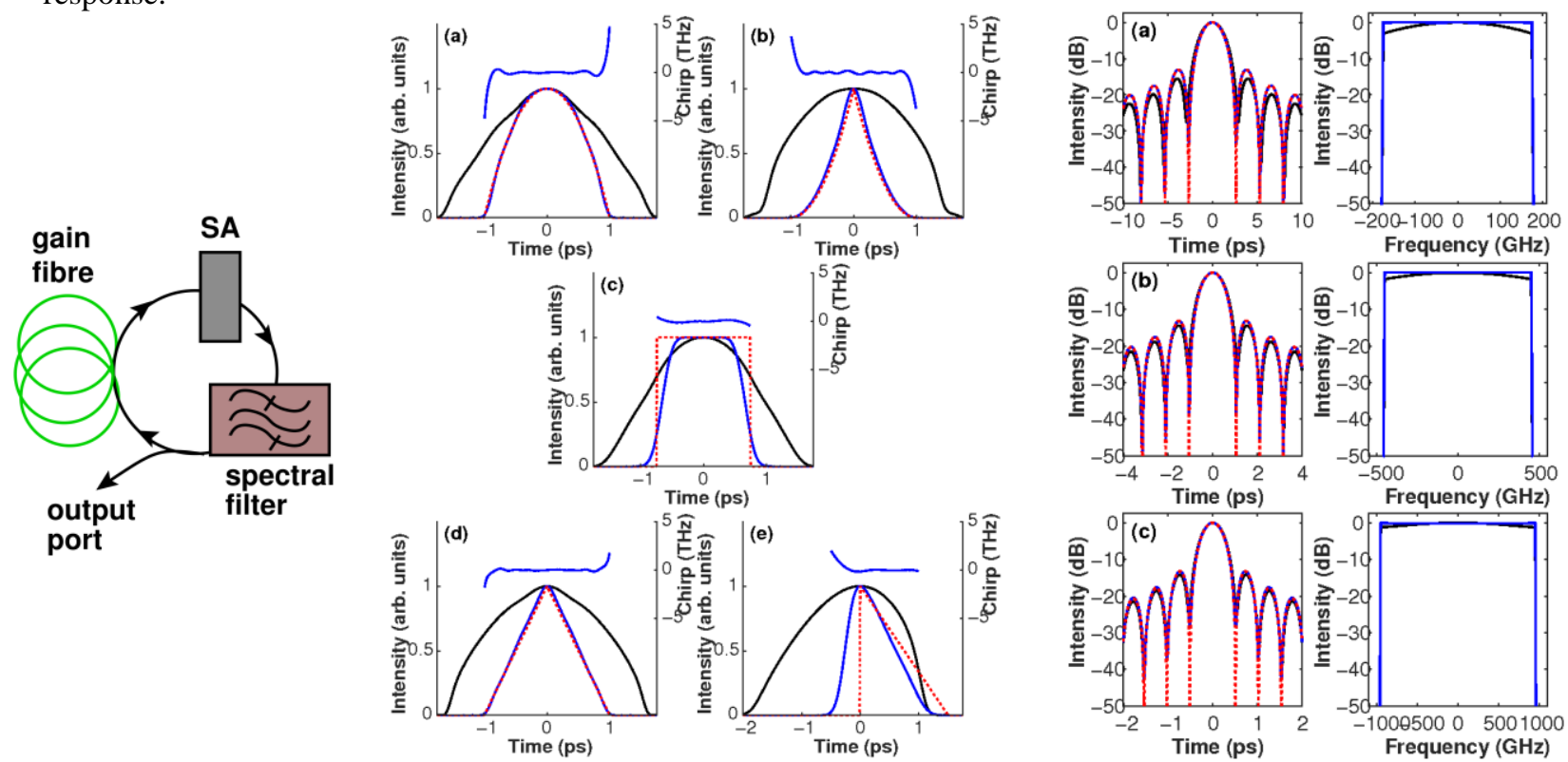

Figure 1. Left: Schematic of the laser. Center: Temporal intensity and chirp profiles of the output pulse from the laser (blue) for a (a) bright parabolic, (b) dark parabolic, (c) flat-top, (d) triangular and (e) saw-tooth impulse response of the spectral pulse shaper. Also shown are the target profiles (red dotted) and the profiles at the exit of the gain fibre (black). Right: Temporal and spectral intensity profiles of the output pulse from the laser for different filter bandwidths: (a) $B=400 \mathrm{GHz}\left(\tau_{p}=2.5 \mathrm{ps}\right)$; (b) $B=1 \mathrm{THz}\left(\tau_{p}=1 \mathrm{ps}\right)$; and (c) $B=2 \mathrm{THz}\left(\tau_{p}=0.5\right.$ $p s)$. The profiles obtained with a flattop spectral pulse shaper (black) are compared to those obtained with a convex Gaussian-top spectral shaper (blue). Also shown are the calculated waveforms (red dotted).

As an illustration of the general pulse-shaping principle described above, we consider here the generation of five different amplitude functions [5], which represent some examples of interesting waveform generations. We use a bright parabolic, a dark parabolic, a flat-top, a triangular and a saw-tooth pulse shaper with the respective functions: $r(t)=\left[1-(t / \tau)^{2}\right]^{1 / 2} \theta(\tau-|t|) ; r(t)=(1-|t / \tau|) \theta(\tau-|t|) ; r(t)=\theta(\tau-|t|) ; r(t)=(1-|t / \tau|)^{1 / 2} \theta(\tau-|t|) ;$ and $r(t)=(1-t / \tau)^{1 / 2}$ for $t$ in $[0, \tau], r(t)=0$ otherwise. Here $\theta(x)$ is the Heaviside function. The pulse solutions obtained for the different amplitude profiles applied to the filter show that the desired pulse shapes are obtained at the output of the laser (middle panel of Fig. 1). The generated pulses are nearly transform-limited. However, as opposed to the chirp profile targeted by the filter, the chirp profile of the generated pulses may exhibit a small ripple. This indicates that generating pulses with a well-defined phase profile by use of the method described here is not as straightforward. The in-cavity pulse evolution (not shown) highlighted the basis of the pulseshaping mechanism being used. The nonlinear evolution in the gain fibre is monotonic with the growth of temporal and spectral widths, while the pulse acquires a positive (normal) chirp. The filter compensates for temporal and spectral broadening and cancels the temporal phase accumulation in the fibre. The calculated $B$ integral and spectral breathing of pulse evolution confirmed that the intended pulse shaping in our laser design is achieved through enhanced spectral filtering of a nonlinearly broadened pulse in the cavity [5].

Now we describe the application of our method for in-cavity pulse shaping to the generation of sinc-shaped optical Nyquist pulses [6], which are of key interest for high-capacity optical communication systems [8] and various other applications. In this case, the amplitude profile $R(f)$ of the filter's spectral response is chosen to have sharply-decaying edges outside its bandwidth and a Gaussian top: $R(f)=\left(\tau_{p} / 2\right) \exp \left(\alpha f^{2}\right)$ for $|f|<1 / \tau_{p}, R(f)=$ $\tau_{p} / 4$ for $|f|=1 / \tau_{p}$, and $R(f)=0$ otherwise, where $B=2 / \tau_{p}$ is the spectral bandwidth, $\tau_{p}$ is the time interval between zero crossings of the corresponding sinc-function-like impulse response and $\alpha$ is an adjustable parameter. For the special value $\alpha=0, R(f)$ is the rectangular spectral profile associated with the Nyquist sinc-function impulse response [8], while $\alpha>0(\alpha<0)$ yields a convex or inverted (concave or conventional) Gaussian top. The solutions plotted in the right panel of Fig. 1 show that the use of the basic rectangular shape for the filter's spectral response already allows us to obtain sinc-shaped pulses at the output of the laser that coincide well with the theoretical ones and feature a spectrum that is fairly close to the ideal rectangular case. When a convex spectral profile is applied to the filter, the correction of the non-perfectly-flattened pulse spectrum after the gain fibre by the filter brings about sinc pulses of enhanced quality and with an almost ideal rectangular spectrum at the laser output. In order to illustrate the flexibility of the proposed scheme in terms of output spectral bandwidth, we have changed the zero-crossing duration parameter $\tau_{p}$ in the filter's impulse response over a wide 
range and assessed the quality of the obtained pulses with a metric quantifying the misfit between the pulse profile and the theoretical sinc function. The results showed that when a flattop spectral profile is applied on the filter the quality of the generated sinc pulses improves with increasing values of the filter bandwidth, which enable increasingly higher values of the nonlinear phase shift accumulated in the fibre. This confirms that the pulse-shaping mechanism of our laser design requires self-phase modulation and gets stronger with increasing pulse intensity and nonlinear phase shift. Sinc-shaped pulses with low misfit values are obtained over the bandwidth range from a few hundred $\mathrm{GHz}$ to a few $\mathrm{THz}$. At smaller bandwidths, in order to enhance the spectral breathing, hence, the filtering process, more nonlinear pulse propagation is necessary in the cavity. To this end, one may either use a longer gain fibre segment or incorporate a short segment of passive nonlinear fibre into the cavity [6]. Our results [6] confirmed that high-quality sinc-shaped pulses are indeed obtained over the bandwidth range from a few tens to a few hundreds of $\mathrm{GHz}$ in this case. On the other hand, when the enhanced spectral filtering strategy is deployed, remarkably low misfit values to a sinc pulse shape are possible over the full bandwidth range being studied $(10 \mathrm{GHz}$ to $3.5 \mathrm{THz})$. This relaxes the need for large nonlinear phase accumulation or, in other words, large spectral broadening in the fibre section to obtain a fairly flat pulse spectrum within the filter bandwidth. Indeed, a higher degree of concavity of the output spectrum from the fibre stemming from a lower extent of nonlinear spectral expansion can be offset by a higher value of the correction factor $\alpha$ (Fig. 1).

\section{DISPERSION-MANAGED VERSATILE ULTRAFAST FIBRE LASER}

Here we review the operation of an ultrafast passively-mode-locked fibre laser, which can be readily and reliably switched among conventional soliton, stretched-pulse and DS mode-locking regimes by changing the dispersion and bandwidth of an in-cavity programmable filter only, while no changes are made to the physical layout of the laser cavity [7]. Figure 2(a) shows a schematic of our passively-mode-locked EDF ring laser. The mode-locking mechanism was nonlinear polarisation rotation, facilitated through the inclusion of a combination of two polarisation controllers (PCs) and an in-fibre polarisation-dependent isolator (PDI). The EDF was $1.2 \mathrm{~m}$ long $\left(\beta_{2}\right.$ $=65.05 \mathrm{fs}^{2} / \mathrm{mm}$ at $1550 \mathrm{~nm}$ ) and was pumped through a 980/1550 wavelength-division multiplexer (WDM) by a 976-nm laser diode. Additional anomalous-dispersion fibre associated with the various components of the laser cavity amounted to a length of $8 \mathrm{~m}$. A programmable liquid-crystal-on-silicon phase and amplitude filter was used to realise different regimes of pulse generation in the cavity. Under mode-locking conditions, the laser operated at a repetition period of $89 \mathrm{~ns}$, which remained constant throughout the experiments described below (since there was no change in the optical length of the cavity). The net dispersion of the cavity was adaptively managed by changing the curvature of the parabolic spectral phase profile of the filter. As pulse formation at net anomalous or slightly normal GVD does not depend on spectral filtering, for such dispersion regimes, the amplitude filtering ability of the filter was not used: we employed the default flat-top filter's shape with the maximum achievable bandwidth. On the other hand, for the operation of the laser in the strong net-normal dispersion regime, the filter was programmed to have a narrow-bandwidth Gaussian spectral profile. The output port was connected by a $3-\mathrm{m}$ long segment of standard telecom fibre to an optical spectrum analyser, an autocorrelator and a fast photo-detector to characterise the pulses.

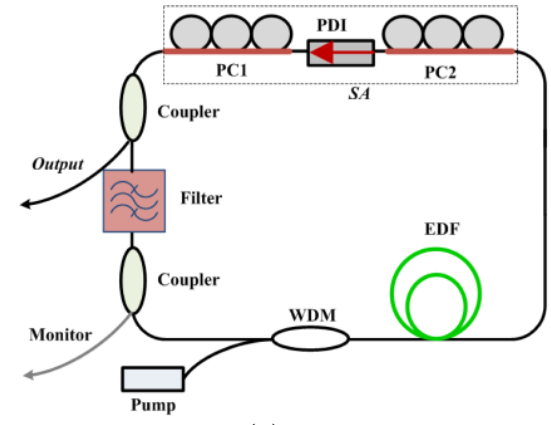

(a)

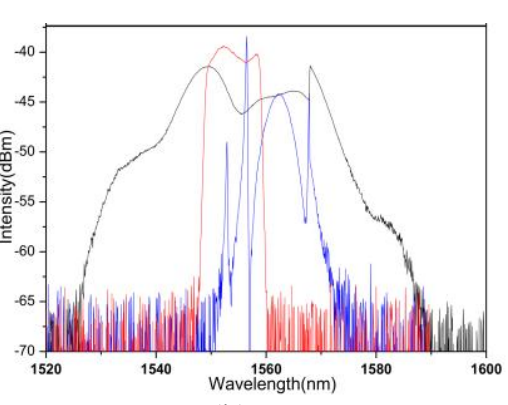

(b)

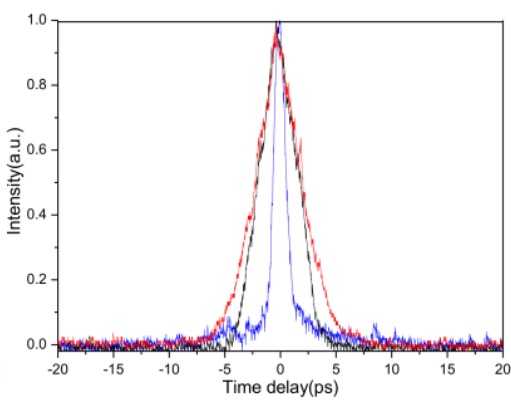

(c)

Figure 2. (a) Schematic of the filter-based dispersion-managed passively-mode-locked EDF laser. Typical (b) measured optical spectra and (c) autocorrelation traces for the output soliton (blue), DM soliton (black) and DS (red).

When there was no dispersion applied on the programmable filter, the cavity had an anomalous net dispersion. By properly adjusting the two PCs in the system, stable mode-locked pulses could be obtained. Typical characterisations of the laser output are given in Fig. 2(b), (c). The optical spectrum profile, centred at $1560 \mathrm{~nm}$ and with a spectral bandwidth at full-width at half-maximum (FWHM) of $4.7 \mathrm{~nm}$, exhibits Kelly sidebands, indicating the fundamental soliton shape of the output pulse. The net dispersion of the cavity, calculated from the 
sidebands, is $-0.3967 \mathrm{ps}^{2}$ (at $1555 \mathrm{~nm}$ ). The measured autocorrelation trace of the pulse corresponds to the FWHM pulse duration $0.8 \mathrm{ps}$ when a hyperbolic secant fit is assumed. This gives a time-bandwidth product (TBP) of 0.46 (at $1560 \mathrm{~nm}$ ), indicating that the pulse is slightly chirped. The soliton-like single-pulse generation regime was observed for a pump power of 20 to $26 \mathrm{~mW}$ and bore an average output power of 0.12 to $0.21 \mathrm{~mW}$ (pulse energy 10.7 to $18.7 \mathrm{pJ}$ ). Next, we applied a dispersion of $0.385 \mathrm{ps}^{2}$ (at $1555 \mathrm{~nm}$ ) on the filter to shift the net cavity GVD close to a zero value. In this case, after adjustment of the PCs, the laser generated pulses (Fig. 2(b), (c)) with a Kelly side-band-free, wide spectrum, which is a signature of the DM soliton operation regime. An even wider output pulse spectrum was obtained by establishing the net GVD to be slightly normal. Singlepulse DM soliton mode locking existed under a pump power of 28.5 to $42 \mathrm{~mW}$ and bore an average output power of 0.24 to $0.41 \mathrm{~mW}$, yielding a pulse energy 21 to $36 \mathrm{pJ}$, which is significantly higher than that of solitonlike mode locking. The autocorrelation trace of the pulse indicates the FWHM pulse duration 2.78 ps when a Gaussian fit is assumed. It is well known that DSs occur in all-normal or strong net-normal dispersion cavities [9]. In our laser design, strong net-normal dispersion can be achieved by configuring the dispersion of the incavity filter; meanwhile, the filter bandwidth also needs to be properly controlled, as DSs require additional amplitude modulation besides SA action. Self-starting mode-locked operation was achieved by adjustment of the PCs with a filter bandwidth of $0.8 \mathrm{THz}$. No stable mode-locked pulses were found for larger bandwidths, consistent with the discussion above. Typical characterisations of the laser output are given in Fig. 2(b), (c) for a net GVD of $0.502 \mathrm{ps}^{2}$ in the cavity. The output pulse spectrum is steep at the edges and has a dip in the top, typical characteristics of DS lasing [9]. The spectral width at FWHM is $9.7 \mathrm{~nm}$. The average output power for the DS mode-locking regime was 0.45 to $0.69 \mathrm{~mW}$ under a pump power of 56.5 to $72 \mathrm{~mW}$, giving the pulse energy 39 to $61 \mathrm{pJ}$. The pulse autocorrelation trace corresponds to the FWHM pulse duration 3.5 ps when a Gaussian fit is assumed. This gives a TBP of 4.21 (at $1555 \mathrm{~nm}$ ), indicating that the pulse is highly chirped inside the cavity [9]. Laser-generated DSs were found to exist in the net cavity dispersion range of 0.247 to $0.505 \mathrm{ps}^{2}$. We have confirmed the different regimes of pulse generation by numerical simulations of the laser [7].

\section{CONCLUSIONS}

We have provided an overview of our recent results and advances in the realisation of pulse shaping in passively-mode-locked fibre lasers by inclusion of an amplitude and phase spectral filter into the laser cavity. We have presented a fibre laser design in which pulse shaping occurs through filtering of a spectrally nonlinearly broadened pulse in the cavity. This strategy of pulse shaping has been illustrated through the numerical demonstration of the laser operation in different pulse-generation regimes, including parabolic, flattop and triangular waveform generations, depending on the amplitude profile of the in-cavity spectral filter. As an application of this method, we have numerically shown the possibility of directly generating sinc-shaped Nyquist pulses of high quality from a laser incorporating a simple flattop spectral filter in the cavity. We have also shown that the use of a filter's profile with a corrective convex top can compensate for the concavity of the nonlinearly broadened pulse spectrum in the fibre, thereby relaxing the need for large nonlinear phase accumulation. The filtering process being used enables the tunability of the spectral bandwidth of the output sinc pulses over a wide range, up to a few THz. Finally, we have reported on a recently developed versatile ultrafast passively-modelocked EDF laser in which the pulse formation mechanism can be flexibly controlled by an in-cavity programmable filter. We have demonstrated that conventional solitons, DM solitons and DSs can be selected and reliably targeted in a single laser by adaptively changing the dispersion and the bandwidth programmed on the in-cavity filter.

\section{REFERENCES}

[1] J. Schröder, T.D. Vo, B.J. Eggleton: Repetition-rate-selective, wavelength-tunable mode-locked laser at up to $640 \mathrm{GHz}$, Opt. Lett., vol. 34, pp. 3902-3904, 2009.

[2] J. Schröder, et al:: Dark and bright pulse passive mode-locked laser with in-cavity pulse shaper, Opt. Express, vol. 18, pp. 22715-22721, 2010.

[3] M. Nakazawa, M. Yoshida, T. Hirooka: The Nyquist laser, Optica, vol. 1, pp. 15-22, 2014.

[4] M.A.F. Roelens, et al:: Dispersion trimming in a reconfigurable wavelength selective switch, IEEE J. Lightwave Technol., vol. 26, pp. 73-78, 2008.

[5] S. Boscolo, et al.: Pulse shaping in mode-locked fiber laser by in-cavity spectral filter, Opt. Lett., vol. 39, pp. 438-441, 2014.

[6] S. Boscolo, C. Finot, S.K. Turitsyn: Bandwidth programmable optical Nyquist pulse generation in passively mode- locked fiber laser, IEEE Photon. J., vol. 7, 7802008(8), 2015.

[7] J. Peng, S. Boscolo: Filter-based dispersion-managed versatile ultrafast fibre laser, Sci. Rep., 2016, In press.

[8] M. Nakazawa, et al: Ultrahigh-speed "orthogonal" TDM transmission with an optical Nyquist pulse train, Opt. Express vol. 20, pp. 1129-1140, 2012.

[9] F.W. Wise, A. Chong, W.H. Renninger: High-energy femtosecond fiber lasers based on pulse propagation at normal dispersion, Laser Photon. Rev., vol. 2, pp. 58-73, 2008. 